\title{
Rhabdomyolysis - A rare cause explained.
}

\author{
Mohamed Baghi ${ }^{1}$, Jassem Sirajudeen ${ }^{1}$, Khaled Alarbi ${ }^{1}$, Vamanjore Naushad ${ }^{1}$, and Nada \\ Benshaban $^{2}$ \\ ${ }^{1}$ Hamad General Hospital \\ ${ }^{2}$ Tripoli Medical Center
}

July 23, 2021

\begin{abstract}
Hypothyroidism causing rhabdomyolysis is a known but an uncommon entity. Hashimoto's thyroiditis causing rhabdomyolysis in the absence of precipitating factors is even rarer. Here we report a 42- year-old previously healthy male with hypothyroidism due to Hashimotos thyroiditis complicated by severe rhabdomyolysis and acute kidney injury.
\end{abstract}

Rhabdomyolysis - A rare cause explained.AuthorsMohamed A Baghi $^{(1)}$ Jaseem Sirajudeen ${ }^{(1)}$ Vamanjore A Naushad ${ }^{(1)}$ Khaled S Alarbi $^{(1)}$ Nada Benshaban $^{(2)}$

Affiliation $^{(1)}$ Department of General Internal Medicine, Hamad Medical Corporation, Doha, Qatar.(2) Department of Critical Care Medicine, Tripoli Medical Center, Libya, Tripoli.Corresponding authorMohamed A Baghi

Post box no 3050

Hamad General Hospital

Hamad Medical Corporation Doha, Qatar Email: mbaghi@hamad.qa Mobile: +97433152064Key Clinical Message

Rarely, hypothyroidism can cause rhabdomyolysis, but it is imperative to maintain a high index of suspicion particularly if no other noticeable causes of rhabdomyolysis can be found.

\section{Abstract}

Hypothyroidism causing rhabdomyolysis is a known but an uncommon entity. Hashimoto's thyroiditis causing rhabdomyolysis in the absence of precipitating factors is even rarer. Here we report a 42- year-old previously healthy male with hypothyroidism due to Hashimotos thyroiditis complicated by severe rhabdomyolysis and acute kidney injury in the absence of precipitating factors. The diagnosis was based on his clinical presentation and laboratory investigations, and he was successfully treated with intravenous fluid therapy and oral levothyroxine.

Keywords- Rhabdomyolysis, hypothyroidism, creatinine kinase, myoglobin

\section{Introduction}

Worldwide, the most common cause of hypothyroidism is iodine deficiency. However, in areas with normal or adequate iodine intake, Hashimoto thyroiditis remains the most common cause of hypothyroidism, with an estimated annual incidence of 0.3-1.5 cases per 1000 persons [1]. Muscular manifestations of hypothyroidism range from muscle weakness, myalgia, muscle cramps with mild to moderately elevated creatinine kinase to more severe forms such as Hoffman's syndrome or rhabdomyolysis [2]. Nevertheless, rhabdomyolysis due to 
hypothyroidism, even in the presence of precipitating factors, is seldomly rare [3]. Here we report an unusual case of rhabdomyolysis due to hypothyroidism without any other associated precipitating factors.

Case presentation A 42-year-old gentleman with no prior medical illness, admitted with the complaints of generalized muscle pain, dry skin, and mild facial puffiness of eight days duration, associated with choking sensation in his throat. The review of systems was negative for fever, hoarse voice, cold intolerance, hair loss, dysphagia, constipation, weight gain, focal limb weakness, or changes in memory. He denied doing strenuous exercise recently, alcohol consumption, trauma, or recent medications use. There was no family history of autoimmune thyroid diseases.

His vital signs were: pulse rate $65 / \mathrm{min}$ (regular), blood pressure $120 / 85 \mathrm{mmHg}$, respiratory rate $19 / \mathrm{min}$, and an oral temperature of $37.1^{*} \mathrm{C}$. Physical examination revealed mild facial puffiness, dry skin, and minimal non-pitting lower limb edema. A small goiter without tenderness or nodule was found on neck examination. The musculoskeletal examination did not show muscle wasting, hypertrophy, or weakness. Other systems examinations were unremarkable.

Laboratory investigations were suggestive of severe hypothyroidism: Thyroid- stimulating hormone (TSH); $>100 \mu \mathrm{IU} / \mathrm{mL}$, (normal range $<4.35 \mathrm{mIU} / \mathrm{L}$ ), Free $\mathrm{T} 4 ;<0.3 \mathrm{ng} / \mathrm{dL}$, (normal range $11-23.3 \mathrm{pmol} / \mathrm{L}$ ) Antithyroid peroxidase antibody titre; $>600 \mathrm{IU} / \mathrm{mL}$, (normal range $<34 \mathrm{IU} / \mathrm{mL}$ ), anti-thyroglobulin antibody $(\mathrm{TgAb})$ titre; $1831 \mathrm{IU} / \mathrm{mL}$, (normal range $<115$ ). Elevated levels of antithyroid peroxidase antibody and anti-thyroglobulin antibody titres were suggestive of Hashimoto's thyroiditis. Serum creatinine kinase (21,644 U/L, normal range 39-308 U/L) and myoglobin $(2,208 \mathrm{ng} / \mathrm{ml}$, normal range $28-72 \mathrm{ng} / \mathrm{ml})$ levels were also raised, Table 1 . This was associated with acute kidney injury with mild elevation of serum creatinine (127 Umol/l). Urine examination was negative for myoglobinuria or hematuria. The daily urine output was normal $(250-300 \mathrm{~mL} / \mathrm{h})$. Electrocardiogram (ECG) and chest x-ray were normal. Transthoracic echocardiography (TTE) showed minimal circumferential pericardial effusion without regional wall motion abnormalities and with a left ventricular ejection fraction of (EF) 58\%. Fiberoptic (flexible) laryngoscopy was normal.A probable diagnosis of hypothyroidism due to Hashimoto's thyroiditis with rhabdomyolysis was made based on the clinical and laboratory parameters. He was treated with intravenous fluids and was started on oral levothyroxine. His symptoms improved with the treatment, and the levels of CK and myoglobin showed a decreasing trend. He was discharged on day four, and on further follow-up, the muscle enzymes showed a further decreasing trend and normalization of renal parameters. Since there was complete resolution of symptoms with the patient returning to his regular day-to-day activities, further workup to exclude muscle diseases was not done.Discussion:

Hypothyroidism manifest with a broad spectrum of clinical features. The Involvement of muscle in various forms is frequently found in hypothyroidism [3]. The muscular symptoms range from stiffness, weakness, myalgia, cramps, pseudohypertrophy, and rhabdomyolysis $[2,4]$. Rhabdomyolysis is a syndrome characterized by muscle necrosis and the release of intracellular muscle constituents into the circulation [4]. The causes of rhabdomyolysis can be due to traumatic or non-traumatic. The various non traumatic causes include heat exhaustion, electrolyte imbalance, seizures, endocrine disorders, infections, and heavy exercise [5]. However, hypothyroidism causing rhabdomyolysis is an infrequent clinical entity and Hashimoto's thyroiditis causing rhabdomyolysis is even rarer, and as per our knowledge, only two cases have been reported so far [7, 8].

Very few cases of hypothyroidism causing rhabdomyolysis has been reported in the literature [9]. Our patient was diagnosed to have rhabdomyolysis without any apparent cause or risk factors. We could not find a definite cause for rhabdomyolysis in the initial evaluation; hence hypothyroidism was considered an underlying etiology, which was confirmed by the laboratory investigations. A review of literature on ten reported hypothyroidism cases causing rhabdomyolysis, it was observed that only four cases had pre-existing hypothyroidism when they presented with rhabdomyolysis. In the remaining six cases the hypothyroidism was diagnosed concurrently with rhabdomyolysis [10]. In cases with rhabdomyolysis due to hypothyroidism, the levels of CK have been reported to be elevated, usually less than ten times the normal range [7, 15]. In the present case, the levels were elevated up to more than 70 times the normal range, which is very unusual in hypothyroidism-induced rhabdomyolysis. The risk of rhabdomyolysis in hypothyroidism is greater in patients 
using statins or after rigorous exercise. However, there was no history suggestive of any risk factors in the present case, including statin use or exercise. The pathophysiology of rhabdomyolysis in hypothyroidism is unclear. Various hypotheses have been postulated, like mitochondrial oxidative metabolism, induction of insulin-resistant state, and decreased muscle carnitine levels, including autoimmune mechanism [11, 13]. Also, deficiency in thyroxine leads to abnormal glycogenolysis and increased triglyceride turnover, thus leading to impairment of muscle function by causing a transition of fast-twitching type two muscle fibers to slow-twitching type one fibers, low myosin ATPase activity, and low ATP turnover in the skeletal muscles [14].

\section{Conclusions:}

Hypothyroidism should be considered as a differential diagnosis of rhabdomyolysis, primarily when no other apparent cause can be found. Early diagnosis and prompt treatment are essential to prevent further serious complications.

Ethical approvalApproval Taken.ConsentApproval was taken from IRB of medical research center of the institution.Author contributionM.Baghi: Writing, Data collection, follow up. J.Sirajudeen: Data collection. V.Naushad: Data collection. K.Alarbi: Data collection. N.Benshaban: Data collection.

Declaration of Competing InterestAll authors declare no conflicts of interest.AcknowledgementOpen access funding provided by Qatar National Library.

\section{References}

1- Vanderpump MP, French JM, Appleton D, Tunbridge WM, Kendall-Taylor P. The prevalence of hyperprolactinaemia and association with markers of autoimmune thyroid disease in survivors of the Whickham Survey cohort. Clin Endocrinol (Oxf). 1998;48(1):39-44. doi:10.1046/j.1365-2265.1998.00343.x 2- Khaleeli AA, Griffith DG, Edwards RH. The clinical presentation of hypothyroid myopathy and its relationship to abnormalities in structure and function of skeletal muscle. Clin Endocrinol (Oxf). 1983;19(3):365-376. doi:10.1111/j.1365-2265.1983.tb00010.x

3- Sindoni A., Rodolico C., Pappalardo M. A., Portaro S., Benvenga S. Hypothyroid myopathy: a peculiar clinical presentation of thyroid failure. Review of the literature. Reviews in Endocrine and Metabolic Disorders. 2016;17(4):499-519. doi: 10.1007/s11154-016-9357-0.

4- Norris FH Jr, Panner BJ. Hypothyroid myopathy. Clinical, electromyographical, and ultrastructural observations. Arch Neurol . 1966;14(6):574-589. doi:10.1001/archneur.1966.00470120006002 5- Leonetti F, Dussol B, Berland Y. Rhabdomyolyse et insuffisance rénale au cours d'une hypothyroïdie [Rhabdomyolysis and kidney failure in hypothyroidism]. Presse Med . 1992;21(1):31-32.

6- Jain S, Bhargava K, Sawlani KK, Daga MK, Gaiha M. Myoglobinuria and transient acute renal failure in a patient revealing hypothyroidism. J Assoc Physicians India . 1999;47(4):444-446. 7- Jobé J, Corman V, Fumal A, Maertens de Noordhout A, Legros JJ. Le cas clinique du mois. Rhabdomyolyse et hypothyroïdie [Rhabdomyolysis and hypothyroidism].Rev Med Liege . 2007;62(7-8):484-486.

8- Nikolaidou C, Gouridou E, Ilonidis G, Boudouris G. Acute renal dysfunction in a patient presenting with rhabdomyolysis due to Hypothyroidism attributed to Hashimoto's Disease. Hippokratia . 2010;14(4):281-283.

9- Gurala D, Rajdev K, Acharya R, Idiculla PS, Habib S, Krzyzak M. Rhabdomyolysis in a Young Patient due to Hypothyroidism without Any Precipitating Factor. Case Rep Endocrinol . 2019;2019:4210431. Published 2019 Dec 3. doi:10.1155/2019/4210431

10- Salehi N, Agoston E, Munir I, Thompson GJ. Rhabdomyolysis in a Patient with Severe Hypothyroidism. Am J Case Rep . 2017;18:912-918. Published 2017 Aug 22. doi:10.12659/ajcr.904691

11- Monzani F, Caraccio N, Siciliano G, Manca L, Murri L, Ferrannini E. Clinical and biochemical features of muscle dysfunction in subclinical hypothyroidism. J Clin Endocrinol Metab . 1997;82(10):3315-3318. doi:10.1210/jcem.82.10.4296 
12- Macleod A, Siddique H, Bashir A, et al. Rhabdomyolysis and acute renal failure due to hypothyroidism. Endocrine Abstracts 2008; 15: P384.

13- Mouzouri H, El Omri N, Sekkach Y, et al. Rhabdomyolyse sévère révélant une myopathie hypothyroïdienne d'origine auto-immune A propos de deux cas [Severe rhabdomyolysis revealing a myopathy linked to autoimmune hypothyroidism]. Ann Endocrinol (Paris) . 2009;70(1):83-86. doi:10.1016/j.ando.2008.05.001

14- Kisakol G, Tunc R, Kaya A. Rhabdomyolysis in a patient with hypothyroidism. Endocr J . 2003;50(2):221223. doi:10.1507/endocrj.50.221

15. Bosch X, Poch E, Grau JM. Rhabdomyolysis and acute kidney injury [published correction appears in N Engl J Med. 2011 May 19;364(20):1982]. N Engl J Med . 2009;361(1):62-72. doi:10.1056/NEJMra0801327

\section{Hosted file}

Laboratory Variables.docx available at https://authorea.com/users/427157/articles/531394rhabdomyolysis-a-rare-cause-explained 